\title{
Submitted: The use of high-frequency ultrasonography in the assessment Accepted: 25.11.2019 of selected female reproductive structures: the vulva, vagina and cervix
}

Published: 31.12.2019

\section{Keywords}

high-frequency ultrasonography, ultrasound, vulva, vagina, cervix

\author{
Marian Stanisław Migda1, Michał Migda'1,2, Rafał Słapa ${ }^{3}$, \\ Robert Krzysztof Mlosek³, Bartosz Migda ${ }^{3}$ \\ ${ }^{1}$ Civis Vita Medical Center Torun, Poland \\ ${ }^{2}$ Clinical Unit of Obstetrics, Women's Diseases and Gynecological Oncology, United District \\ Hospital, Collegium Medicum University of Nicolaus Copernicus in Torun, Torun, Poland \\ ${ }^{3}$ Department of Diagnostic Imaging, Second Faculty of Medicine with the English Division \\ and the Physiotherapy Division, Medical University of Warsaw, Warsaw, Poland
}

Correspondence: Marian Migda, Warszawska 20, 87-100 Toruń;

e-mail:marian.s.migda@gmail.com

DOI: $10.15557 / J o U .2019 .0039$

\begin{abstract}
Introduction: High-frequency ultrasonography enables visualization the layered structure of the skin and shows the epidermis, dermis, subcutaneous tissue and skin appendages: hair follicles, sebaceous glands, sweat glands and blood vessels. The aim of this study was to apply high-frequency ultrasonography to evaluate the structure of the vulva, vagina and cervix, and to describe their anatomy in ultrasound. Material and method: The examinations were conducted with the use of high-frequency probes: DermaView $48 \mathrm{MHz}$ and Episcan $50 \mathrm{MHz}$, by three operators experienced in performing classical ultrasound scans (with 30-, 10- and 9 -year experience). The study involved 50 women aged $20-80$ years who reported for a periodical gynecological check-up and presented no symptoms within the reproductive system. Results: In this study, the individual layers of the skin in the area of the mons pubis, labia majora (together with hair follicles and sweat glands) and labia minora were successfully visualized in all the patients. The subepidermal low-echogenicity band was seen in the area of the mons pubic and labia majora in 40 cases. This concerned $100 \%$ of women who underwent cosmetic skin treatments. In all the patients, HFUS demonstrated the layered structure of the anterior and posterior vaginal walls, the walls of the urinary bladder and rectum, layers of the vaginal portion of the cervix and the external opening of the cervix. Conclusions: High-frequency ultrasonography offers new quality of vulvar, vaginal and cervical imaging. It can be used for evaluation of the vulva and vagina, and confirms their layered structure. It also enables accurate measurements of the thickness of the vulvar epidermis and dermis as well as the thickness of the vaginal walls.
\end{abstract}

\section{Introduction}

Ultrasound (US) is an imaging modality used in various fields of medicine for diagnostic purposes, for guidance during interventional procedures and for assessment of treatment effects. The frequencies used in gynecological and obstetric ultrasound range from $3-5 \mathrm{MHz}$ and $4-8 \mathrm{MHz}$ in transabdominal probes to $5-11 \mathrm{MHz}$ and $6-9 \mathrm{MHz}$ in endovaginal and transrectal probes.

Conventional ultrasound in the frequency range 7-15 MHz enables imaging to the depth of $70 \mathrm{~mm}$ with axial and spatial resolution at the level of $0.2 \mathrm{~mm}^{(1)}$. In dermatology, this technique appeared in 1979 when Alexander et al. used it to 
measure the $\operatorname{skin}^{(2)}$. In the subsequent years and at present, ultrasound techniques have been observed to advance rapidly, and new high-frequency transducers offer highly detailed images. That is why, apart from conventional ultrasound, high-frequency ultrasonography (HFUS), with the frequency range of 20-50 MHz, can be used in the diagnosis of skin lesions, offering new quality of ultrasound in dermatology $y^{(3,4)}$. The images obtained at this frequency range are characterized by superior axial and lateral resolution, where the values for $20-25 \mathrm{MHz}$ are 80 and $200 \mu \mathrm{m}$, respectively. This, however, causes certain limitations in the form of a reduction of the depth of penetration, in this case, to $8 \mathrm{~mm}^{(1)}$. An advantage of this method, which has been confirmed in examinations of skin lesions, is "histological" assessment and identification of the consecutive skin layers, measurement of the layers in millimeters and determining the echogenicity in the number of pixels ${ }^{(5)}$. High-frequency ultrasonography has proven useful in dermatology, esthetic medicine and cosmetology, which has been confirmed in various publications ${ }^{(6-10)}$.

The application of this imaging modality in gynecology was described for the first time by Migda et al., who evaluated the structure of the vulvar skin and described the anatomy in $\operatorname{HFUS}^{(11)}$.

The aim of this report is to present ultrasound description of the vulvar skin, vaginal structures and cervix in HFUS.

\section{Material and methods}

The study was designed and performed in cooperation with the Department of Medical Imaging, Second Faculty of Medicine, Medical University of Warsaw. The pilot study was approved by the Ethics Committee of the Medical University of Warsaw (No KB/220/2012), and all the patients expressed informed consent to participate.

HFUS was conducted by three operators experienced in conventional ultrasound (with 30-, 10- and 9-year experience).

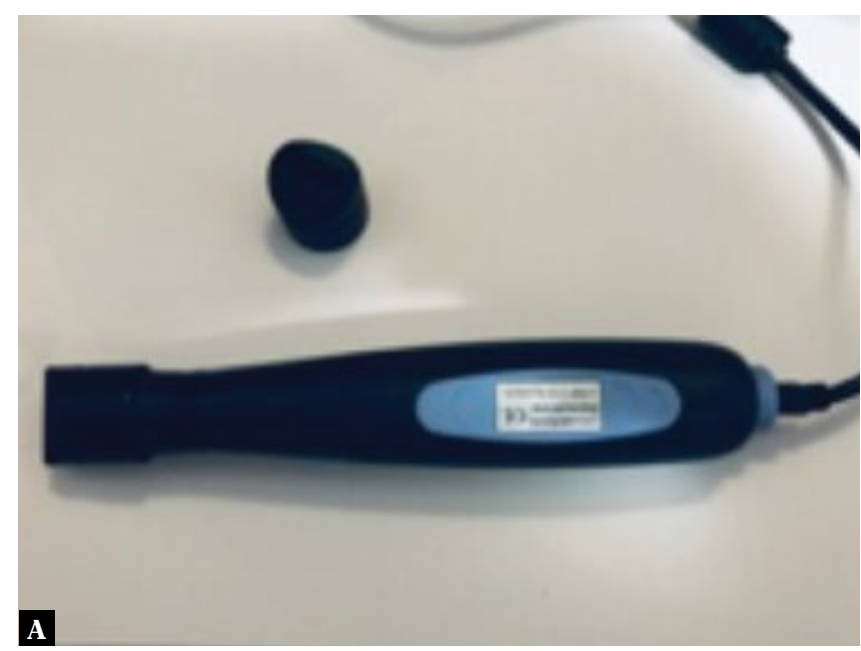

The study involved 50 women aged $20-80$ years who reported for a periodical gynecological check-up and presented no symptoms within the reproductive system. Patients reporting symptoms within the reproductive system or those with pathological changes found on pelvic examination or transvaginal ultrasound were excluded. The patients underwent a pelvic examination and transvaginal ultrasound using RIC 5-9 D transducer and Voluson E-8 machine. Upon receipt of written consent, high-frequency ultrasonography (HFUS) of the vulva, vagina and cervix was performed.

\section{Equipment}

Two high-frequency scanners were used in the study: Episcan (Longport International, Ltd., USA) with a mechanical transducer of $50 \mathrm{MHz}$ and DermaView (Dramiński S.A., Poland) with a mechanical transducer of $48 \mathrm{MHz}$.

Due to the shape and structure of both transducers, the transducers from both of the Episcan and DermaView machines were used for vulvar examination, while vaginal structures and the cervix were evaluated using the transducer of the DermaView scanner (Fig. 1).

\section{Vulvar structures: protocol and scanning technique}

The evaluation of the vulvar structures in HFUS was conducted in accordance with the protocol described below with the use of the Episcan machine. The following structures were evaluated in the consecutive order: the mons pubis, the labia majora and minora, the clitoris, the greater vestibular glands, the lesser vestibular glands (so-called Skene's glands) and the vulvar vestibule ${ }^{(12)}$. All the structures were visualized at the right angle in relation to the surface of the skin and, if possible, in available perpendicular and oblique planes. Three best sections were archived on a working station in the form of static images, and then analyzed.

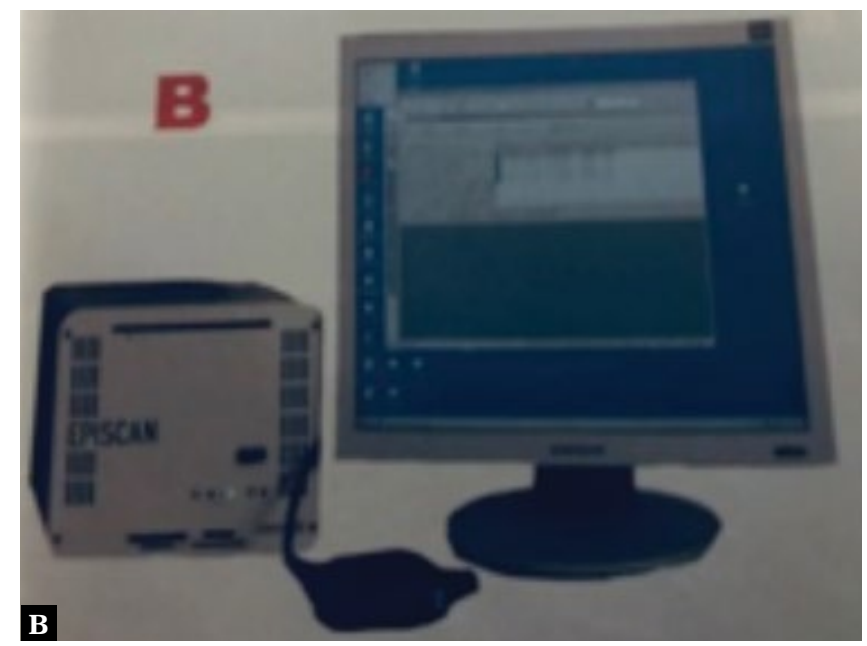

Fig. 1. High-frequency ultrasound. A. DermaView with a mechanical transducer of $48 \mathrm{MHz}$ and $\mathbf{B}$. Episcan with a mechanical transducer of $50 \mathrm{MHz}$ 

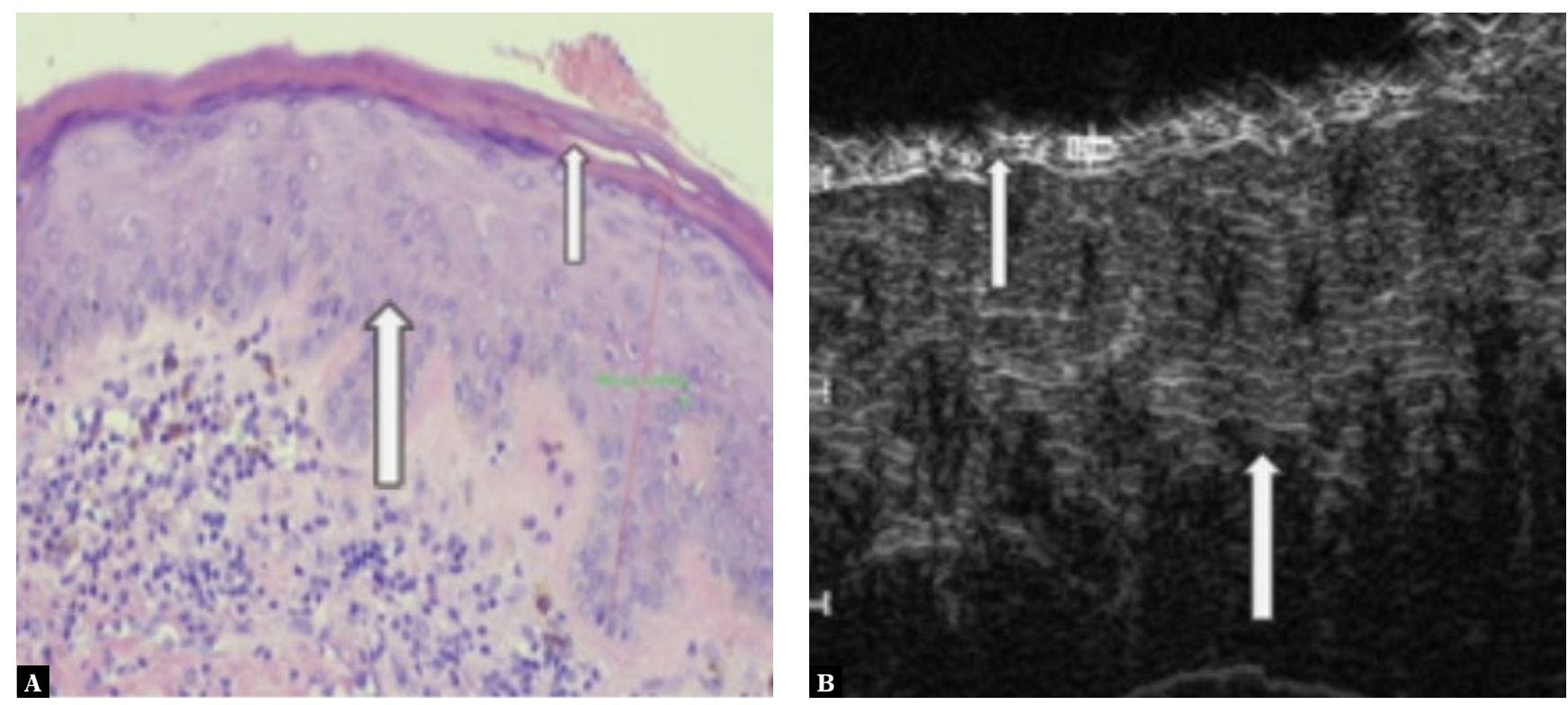

Fig. 2. Histopathological image of the skin of the pubic mound (A) with the corresponding HFUS image (B). Epidermis (small arrow), dermis (large arrow), Episcan, transducer of $50 \mathrm{MHz}$
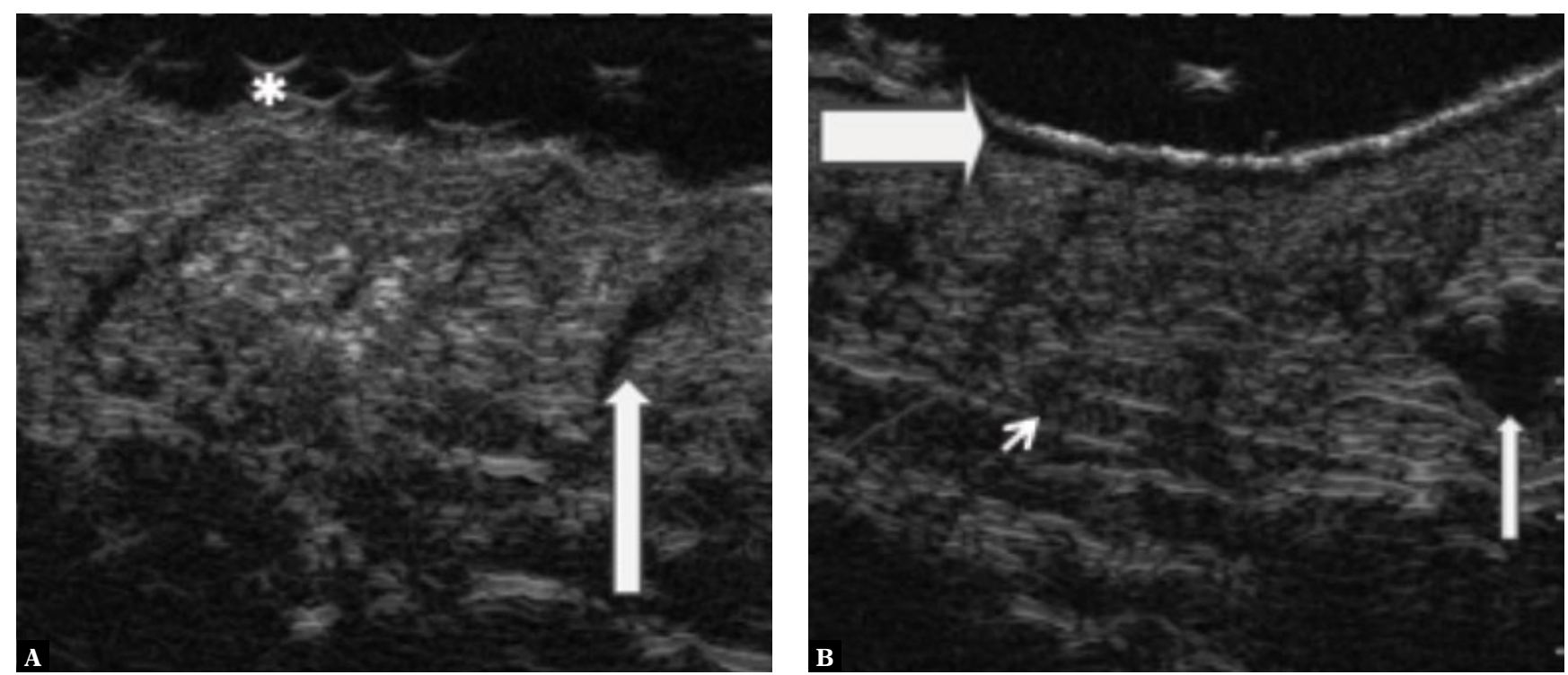

Fig. 3. A. HFUS of the skin of the labia majora and minora with visible hair (asterisk) and marked hair follicle (large arrow). B. An image of the labia majora with removed hair and a visible subepidermal low-echogenicity band (SLEB, thick arrow), sweat gland (arrow head) and blood vessel (small arrow)

The examination of the vulvar structures was conducted on a gynecological chair in the lithotomy position, upon placement of gel onto the skin of the examined area. The patients were asked to remove hair from the pubic region and labia on the day preceding the examination in order to minimize the number of artefacts generated by hair. While scanning, the transducer was moved perpendicularly to the examined structure, i.e. to the mons pubis, labia majora and minora, clitoris. When evaluating the labia majora and minora, an additional thick layer of gel was applied, if necessary, in order to increase the distance to the examined structure and to improve image quality. The best sections of the epidermis and dermis were archived using the software of the Episcan Ultrasound
Scanner Version 4.0.0.030 (Longport International, Ltd.), provided by the manufacturer.

Vulva: mons pubis (Fig. 2), labia majora (Fig. 3), external urethral orifice (Fig. 4), Hart's line (Fig. 5), labia minora imaged with a "gel pad" (Fig. 6), clitoris (Fig. 7).

\section{Vaginal structures: protocol and scanning technique}

The evaluation of the vaginal structures in HFUS was conducted in accordance with the protocol described below with the use of the transducer of the DermaView machine. 

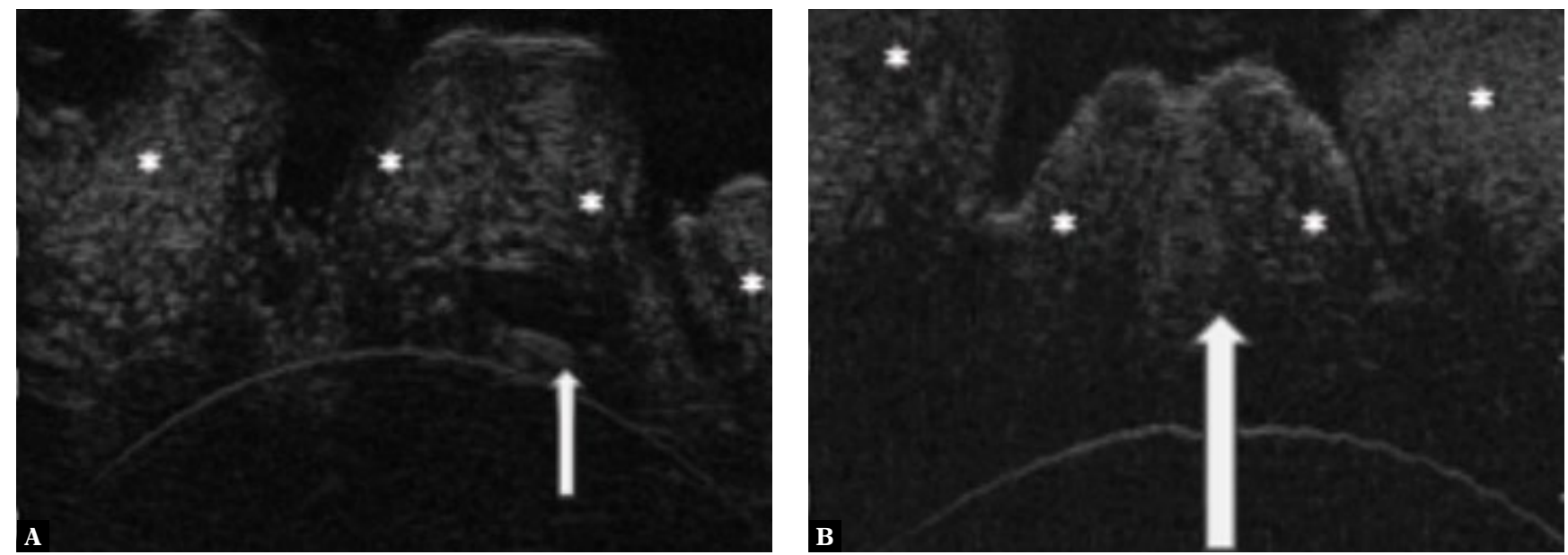

Fig. 4. A. HFUS of the external urethral orifice in a multiparous woman with a visible periurethral Skene's gland (small arrow), and B. the external urethral orifice in a primiparous woman (large arrow) with visible periurethral folds (asterisk)

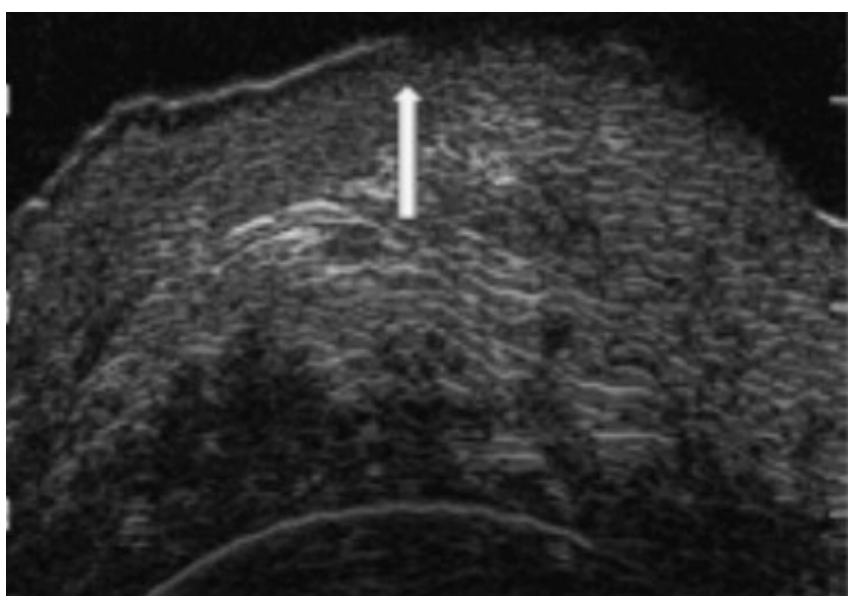

Fig. 5. HFUS. Interepithelial boundary - Hart's line (arrow)

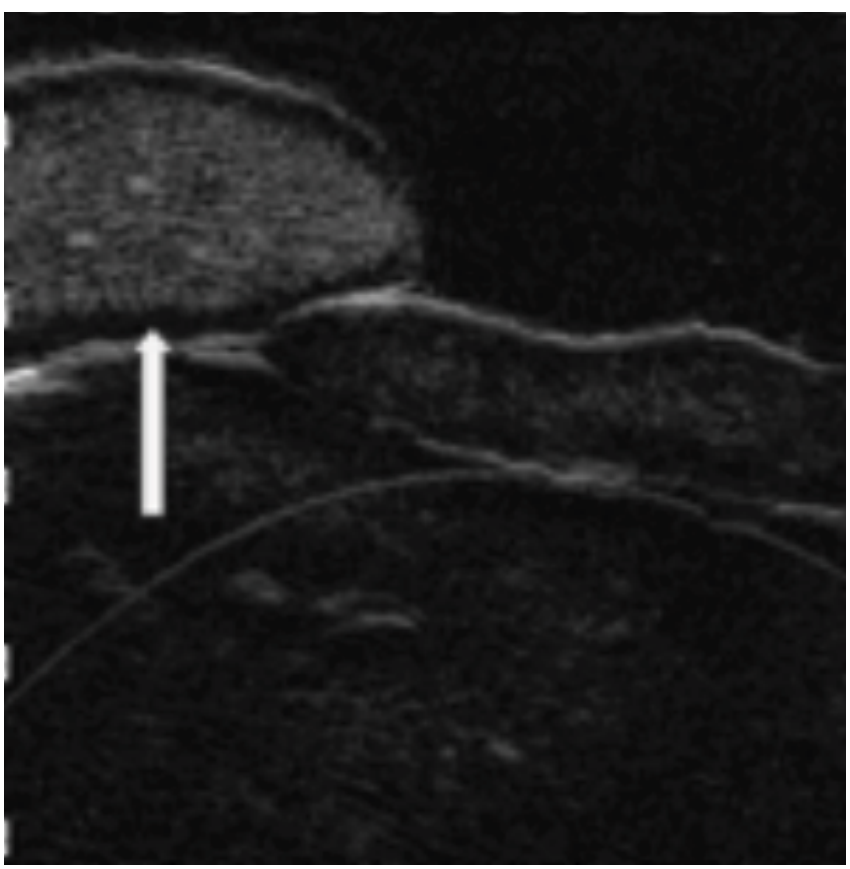

Fig. 7. Clitoris with the clitoral hood in HFUS (arrow)

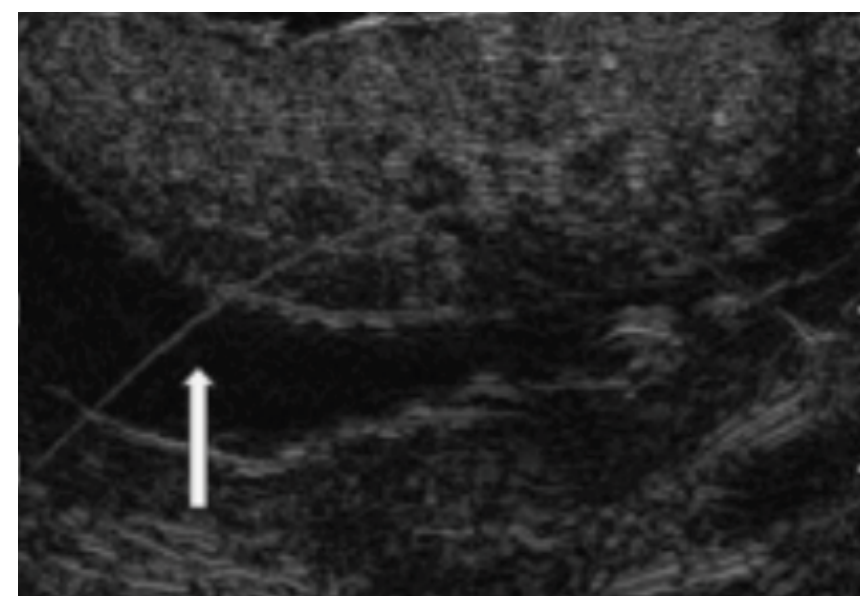

Fig. 6. HFUS of the labia minora with a "gel pad" (arrow)

The following structures were evaluated consecutively: the anterior vaginal wall and the adjacent fragment of the urinary bladder, the posterior vaginal wall, the adjacent rectal wall and the external vaginal opening. Three best sections were archived on a working station in the form of static images or shadow loops, and then analyzed. In order to minimize errors, each structure was evaluated three times, and the best section was used for analysis.

The examination was performed in the lithotomy position on a gynecological chair. The transducer was covered with a shield for endocavitary probes and introduced to the vagina along its long axis. When the cervix was visualized, the transducer was moved upwards towards the anterior vaginal wall to obtain its image along with the image of the posterior wall of the urinary bladder. Subsequently, the transducer was moved posteriorly towards the posterior vaginal wall and the adjacent rectum. Afterwards, the transducer was withdrawn towards the external vaginal opening. The evaluation of each wall (anterior and posterior) was performed at three levels: at the level of the cervix and anterior vault (anterior vaginal wall), at the center of the vagina and before the external vaginal opening: the area of the urethra and the posterior vaginal vault (posterior vaginal wall), and before the external opening: 


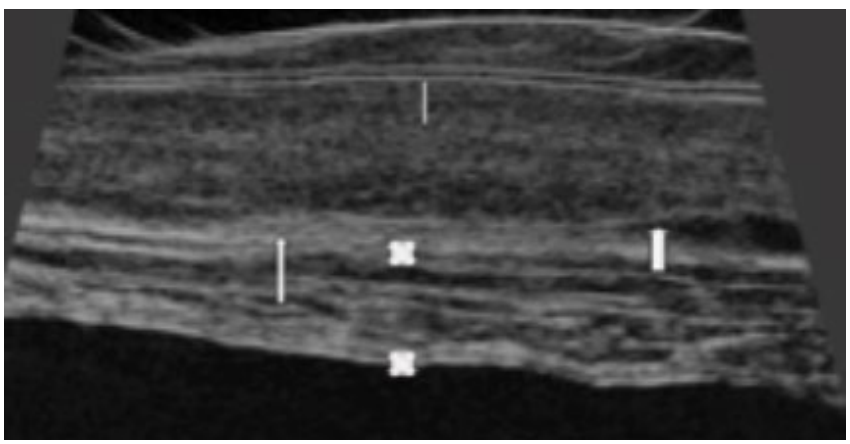

Fig. 8. HFUS of the anterior vaginal wall (small arrow: mucosa; thick arrow: muscle layer; long arrow: adventitia) with the adjacent wall of the urinary bladder (area between asterisks)

the area of the anus. During high-frequency examinations, the structure and elements of the anterior and posterior vaginal walls were assessed (Fig. 8, Fig. 9) and transperineally (Fig. 10 A), transabdominally (Fig. $10 \mathrm{~B}$ ).

\section{Cervix: protocol and scanning technique}

The evaluation of the cervix in HFUS was conducted with the use of the transducer of the DermaView machine.

The examination was performed in the lithotomy position on a gynecological chair. The transducer was covered with a shield for endocavitary probes and introduced to the vagina along its long axis until its head rested on the edge of the anterior cervical lip. The transducer was then moved in the sagittal and transverse planes to obtain the images of the anterior lip, external opening and posterior lip of the cervix (Fig. 11, Fig. 12, Fig. 13, Fig. 14).

The visualization of the cervix in transabdominal US with the filled urinary bladder is presented in Fig. 15.

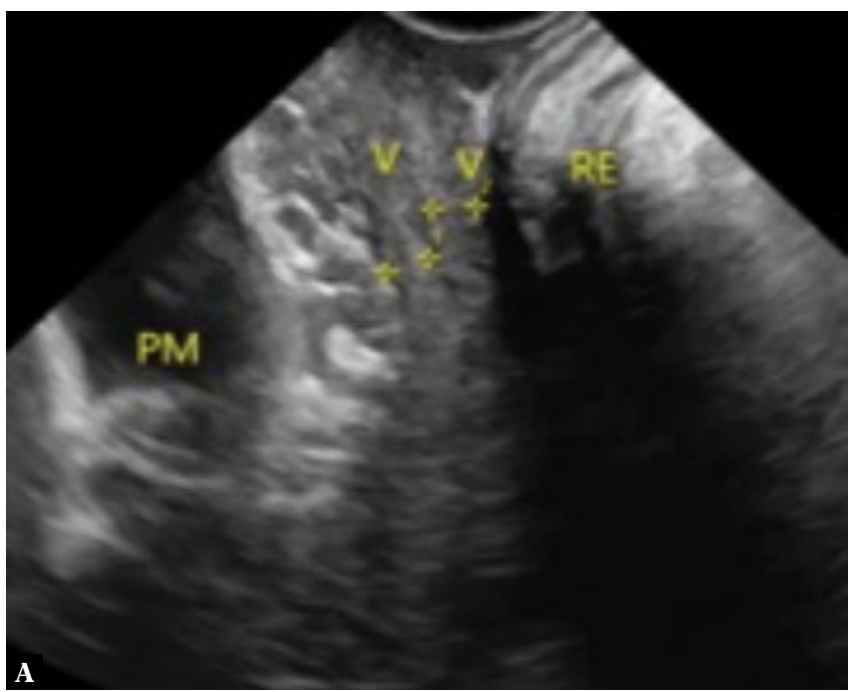

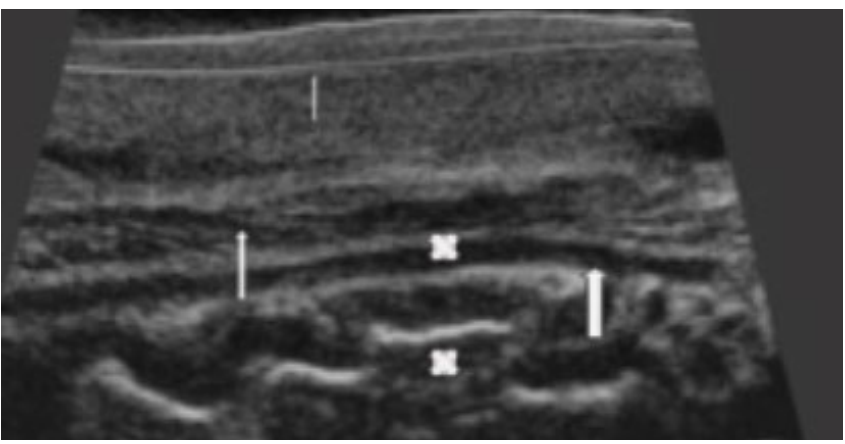

Fig. 9. HFUS of the posterior vaginal wall with the adjacent rectal wall. Mucosa of the posterior vaginal wall (arrow), muscle layer (large arrow), adventitia (thick arrow). The layered structure of the intestine is visible between asterisks

The visualization of the cervix in transvaginal US: the external opening presents itself on the right of the cervical canal, whilst the urinary bladder should be visible just above the cervix. An ideal image is characterized by the visualization of the cervical canal from the internal opening to the external opening with the anteflexed uterus, where the cervical canal is at the near-to-right angle in relation to the axis of the vagina ${ }^{(13-15)}$ (Fig. 16).

\section{Results}

In this study, the individual layers of the skin in the area of the mons pubis, labia majora (together with hair follicles and sweat glands) and labia minora (Fig. 2 B, Fig. 3 A and B, Fig. 6) were successfully visualized in all the patients with the use of mechanical probes of the Episcan and DermaView scanners.

The subepidermal low-echogenicity band (SLEB) was seen in the area of the mons pubic and labia majora in 40 cases. This concerned $100 \%$ of women who underwent cosmetic

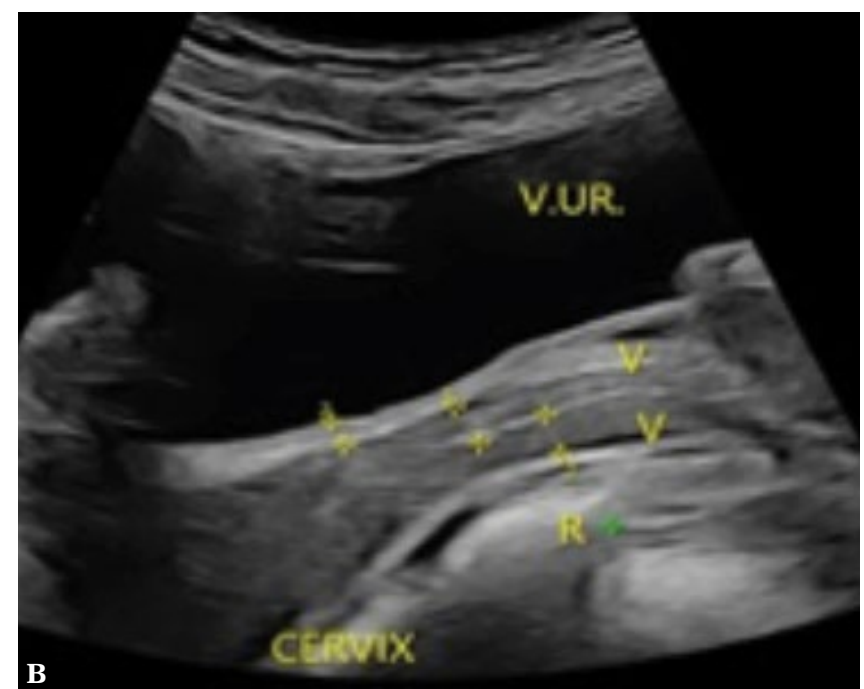

Fig. 10. Vaginal ultrasound. A. Transperineal examination. B. Transabdominal examination. PM, V.UR-urinary bladder, V-vaginal wall, $R, R E$ - rectum, cervix-cervix uteri. Markers show the anterior and posterior vaginal walls 


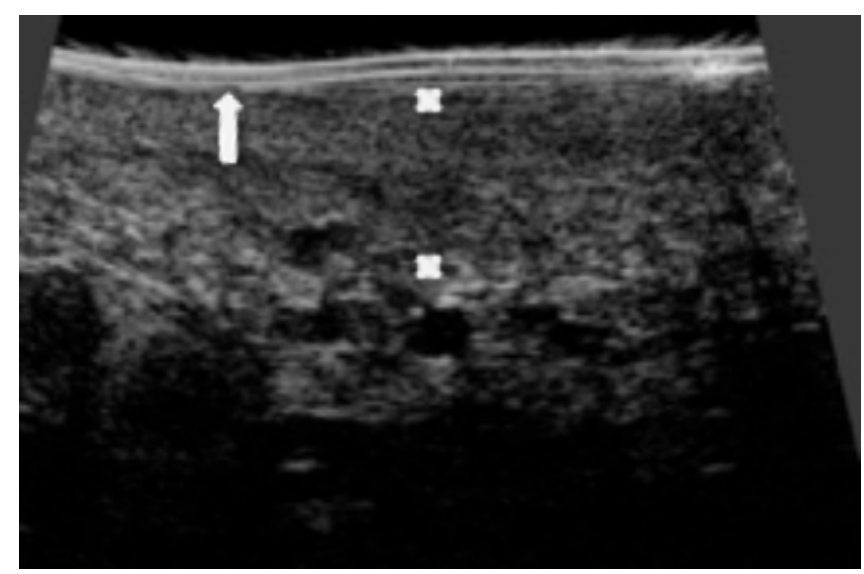

Fig. 11. Echostructure of the vaginal portion of the cervix in HFUS with a visible muscle layer (between asterisks) and blood vessels located underneath. The non-keratinizing stratified squamous epithelium (arrow) is covered with reflections from the transducer shield in HFUS

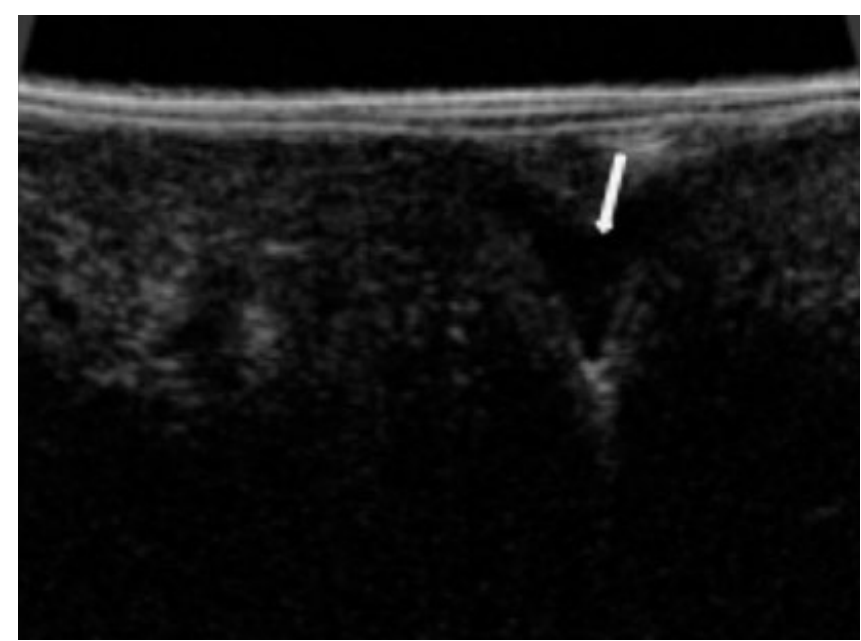

Fig. 12. External opening of the cervix in HFUS (arrow)

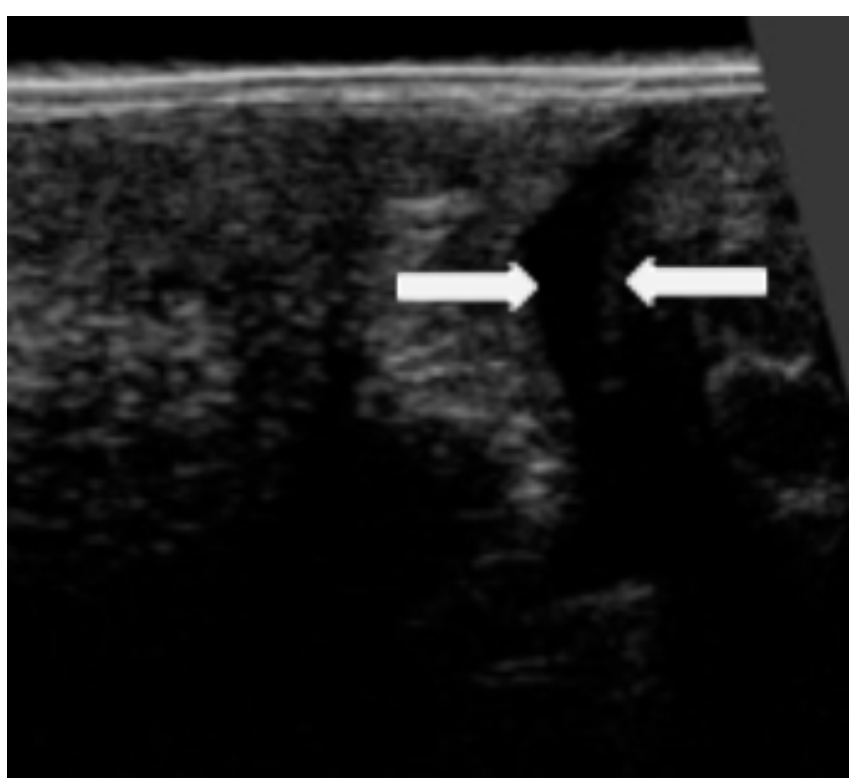

Fig. 13. Cervical canal in HFUS (between arrows) skin treatments. In the remaining 10 cases, where hair was not removed, SLEB was not visible.

The Hart's line, i.e. the interepithelial boundary, clitoris and the area of the urethral orifice with periurethral folds were visible in all the examined women irrespective of the transducer used for examination (Fig. 4, Fig. 5, Fig. 7).

HFUS successfully demonstrated the layered structure of the anterior and posterior vaginal walls and the walls of the urinary bladder and rectum, layers of the vaginal portion of the cervix and external opening of the cervix in all the patients.

\section{Discussion}

HFUS offers broader diagnostic possibilities in vulvar imaging as it shows the layered structure of the skin, epidermis, dermis and skin appendages, and enables one to measure the thickness of the skin and epidermis. At present, the evaluation of vulvar lesions is made by inspection, palpation, vulvoscopy, swabs for microbiological analysis and biopsy ${ }^{(11)}$. In HFUS of the vulva, attention should be paid to certain obstacles, such as the presence of hair which produces artefacts (Fig. $3 \mathrm{~A}$ ) in the form of acoustic shadows or a reduction of contrast between the epidermis and dermis. That is why hair should be removed from the mons pubis and labia majora prior to scanning. As a result of the local skin irritation and subepidermal reaction, SLEB may appear, as shown in this material. The band can also be noticed in other clinical situations, e.g. in dermatitis, eczema or psoriasis $^{(12,13,14)}$. The visualization of the labia minora may pose another problem due to their size or attachment to the labia majora. To improve their imaging, gel should be applied between the labia majora and minora, thus creating a "gel pad" (Fig. 6) that acts as an acoustic window and improves the assessment of this structure.

The vagina is a muscular and membranous canal with the length of $8 \mathrm{~cm}$. It begins with the vaginal vestibule and runs between the posterior wall of the urinary bladder and urethra anteriorly and rectum posteriorly. The upper segment of the vagina is closed with the vaginal portion of the cervix, forming the anterior and posterior vaginal vault ${ }^{(15)}$.

The histological structure of the vagina is as follows: the mucosa directed towards the vaginal lumen, underlining muscle consisting of two layers of smooth muscle: the thicker longitudinal layer and the thinner circular layer, as well as an outer connective tissue layer called the adventitia ${ }^{(16-18)}$. HFUS shows the layered structure of the vagina from the lumen onwards: the mucosa directed towards the vaginal lumen, a muscle layer that cannot be further differentiated into longitudinal or circular, and the connective tissue of the adventitia, which is followed by the posterior wall of the urinary bladder behind the anterior vaginal wall or by the anterior rectal wall behind the posterior vaginal wall (Fig. 8 and Fig. 9). The ultrasonographic evaluation of the vagina and the distal aspect of the cervix is limited with various sonographic and anatomic artefacts. The sonographic 


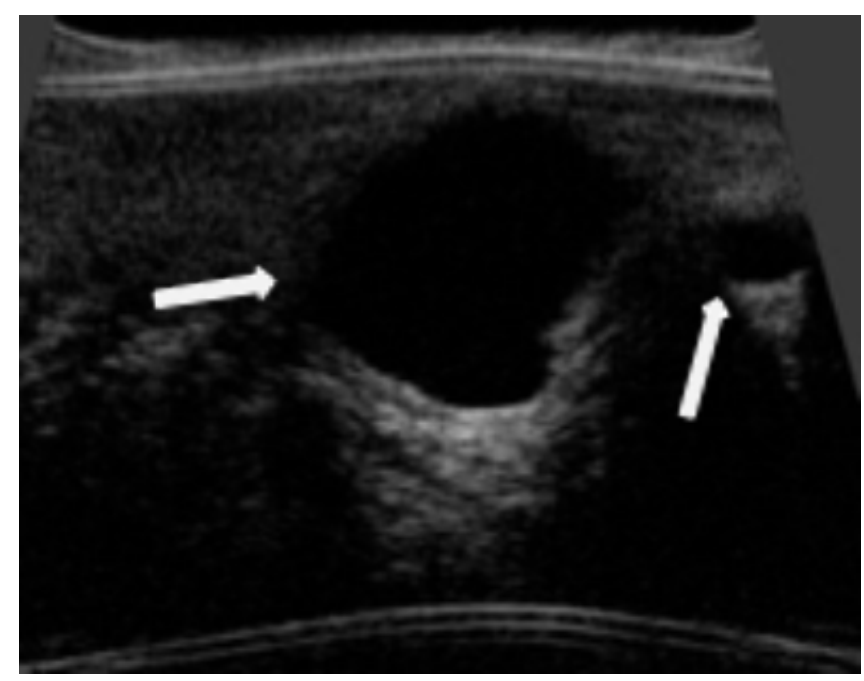

Fig. 14. Nabothian cysts in the cervix in HFUS (arrows)

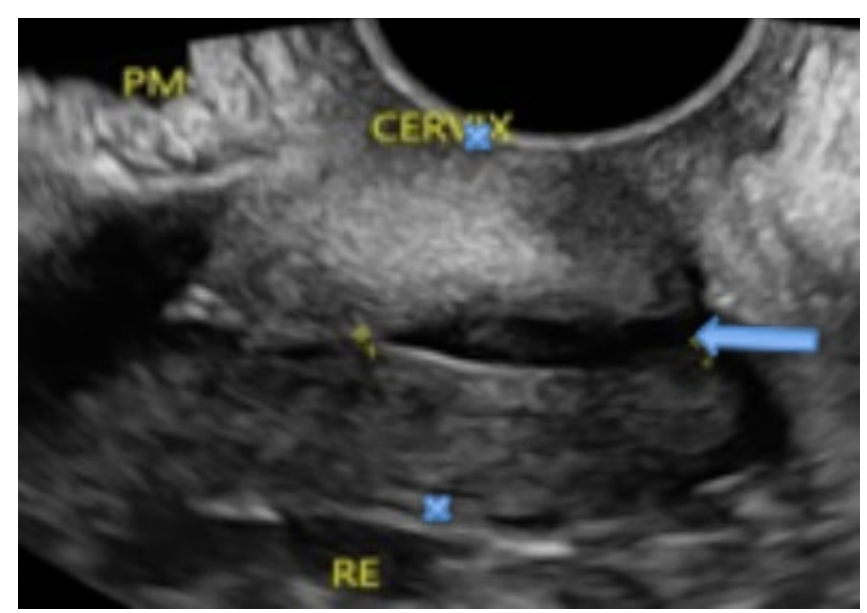

Fig. 15. Cervix in transabdominal ultrasound (arrow: external cervical opening; between asterisks: cervix), PM: urinary bladder

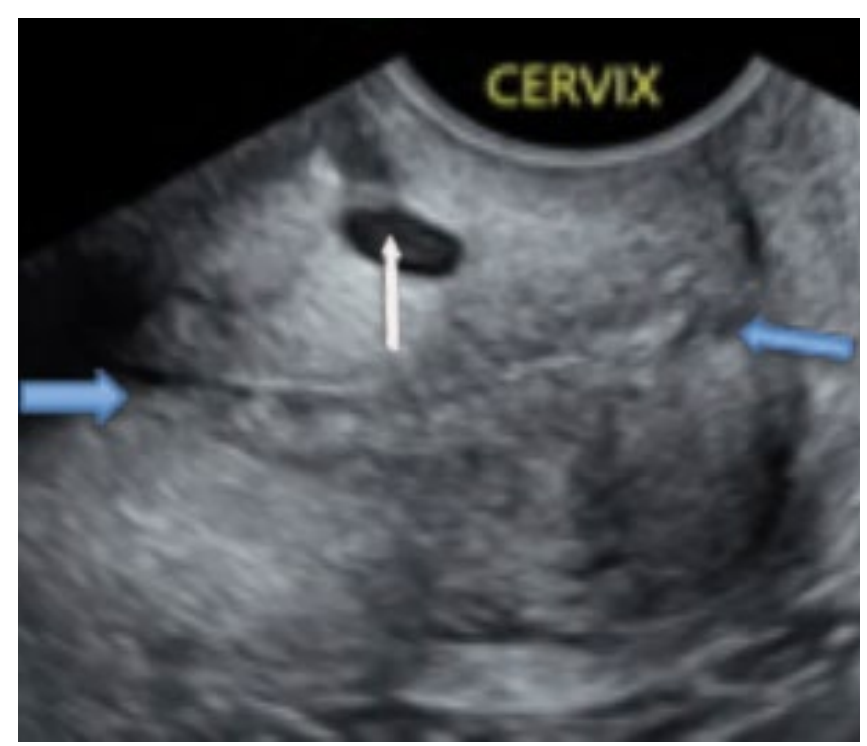

Fig. 16. Cervix in transvaginal ultrasound, the internal and external opening (large arrows); the cervical canal visible between arrows, small arrow: Nabothian cyst factors restricting the visualization of the vaginal walls are: too deep placement of the transducer reaching the vaginal vault ${ }^{(19)}$, poor contrast resolution of the boundaries between the cervix and pathological lesions in the vagina, and deformation of the cervix and vagina induced by the pressure of the transducer. The anatomic factors that have an impact on the evaluation are: variable position of the cervix in relation to the vagina depending on the position of the uterus, the degree to which the urinary bladder and rectum are filled, and tension of the pelvic diaphragm depending on the level of patient relaxation ${ }^{(20)}$. At present, vaginal imaging can be improved by applying other techniques of evaluation. Transabdominal ultrasound with the filled bladder ${ }^{(21,22)}$ allows the presentation of advanced pathological lesions that arise from the cervical canal or vaginal portion of the cervix $^{(23)}$. In transvaginal US, usually in the sagittal plane, where the end of the transducer touches the anterior edge of the cervix and the urinary bladder is visible just above the cervix, the internal opening is on the right side of the cervical canal ${ }^{(18,23)}$, and the evaluation of the unchanged vaginal walls is limited and uncertain. Transperineal ultrasound offers more complete visualization of the lower part of the cervix and the vagina as well as lesions in this area that could be overlooked in a standard cervical and vaginal examination; in this scan, the transducer is fixed in the vaginal vestibule ${ }^{(17)}$. During sonovaginography, the evaluation of the vagina and cervix is conducted after filling the vagina with US gel and after assessment of these structure in 2D US (B-mode) and MRI (magnetic resonance imaging $)^{(22,24)}$. Additionally, it is possible to visualize fistulae and defects of the vagina after gel application and assessment in 3D US ${ }^{(22,25)}$. The number of the presented methods shows how important imaging is for the evaluation of vaginal pathologies, which may be overlooked during a conventional examination.

HFUS of the vagina has certain method-related limitations. First of all, the transducer used is not a typical endovaginal probe (Fig. $1 \mathrm{~A}$ ), the contact of the transducer head with the examined structure (vaginal wall) in the area of the vaginal vault is not fully perpendicular, which affects the quality of obtained images, and the visualization of the lateral walls of the vagina is difficult due to anatomic restrictions that prevent proper positioning of the transducer in the evaluated region; the transducer should be moved slowly over the examined area. Second, the adherence of the transducer head covered with a US shield to the vaginal portion of the cervix restricts the evaluation of its first layer, i.e. the ectocervix, due to reflections of ultrasounds from the shield and the epithelium. The attempts to keep more distance between the transducer head and the vaginal portion of the cervix by applying a layer of US gel have not had a significant impact on imaging improvement due to a high number of artefacts generated by air bubbles contained in the conjugation material applied in this way.

HFUS is a supplementation of vulvar, vaginal and cervical imaging and offers new quality for the morphological evaluation of the investigated structures, presenting more details. However, this is obtained at the expense of the depth of penetration and the size of the evaluated vulvar region. 
HFUS enables measurement of the thickness of the vulvar epidermis and dermis and thickness of the vaginal wall. It also offers new possibilities in assessment and measurement of the evaluated structures. This implies that the described method is interesting from the diagnostic point of view and requires further investigation.

\section{Conclusions}

High-frequency ultrasonography (HFUS) is characterized by better resolution, both axial and lateral, but also has limitations in the form of lower depth of penetration. An advantage of this method, which has been confirmed in examinations of skin lesions, is "histological" assessment and identification of the consecutive skin

\section{References}

1. Migda B, Mlosek RK, Słapa R: Ultrasonografia klasyczna i wysokich częstotliwości w diagnostyce chorób i patologii skóry. Ultrasonogr 2011; 11: 67-73.

2. Alexander H, Miller DL: Determining skin thickness with pulsed ultra sound. J Invest Dermatol 1979; 72: 17-19.

3. Scola N, Goulioumis A, Gambichler T: Non-invasive imaging of middermal elastolysis. Clin Exp Dermatol 2011; 36: 155-160.

4. Jasaitiene D, Valiukeviciene S, Linkevicitute G, Raisutis R, Jasiuniene E, Kazys R: Principles of high-frequency ultrasonography for investigation of skin pathology. J Eur Acad Dermatol Venereol 2011; 25: 375-382.

5. Dańczak-Pazdrowska A, PolańskaA, Silny W, Sadowska A, OsmolaMańkowska A, Czarnecka-Operacz M et al.: Seemingly healthy skin atopic dermatitis: observation with use of high-frequency ultrasonography, preliminary study. Skin Res Technol 2012; 18: 162-167.

6. Kleinerman R, Whang TB, Bard RL, Marmur ES: Ultrasound in dermatology: principles and application. J Am Acad Dermatol 2012; 67: 478-487.

7. Mlosek RK: Obrazowanie skóry i tkanki podskórnej za pomocą ultrasonografii klasycznej oraz ultrasonografii wysokiej częstotliwości i jej przydatność w kosmetologii i medycynie estetycznej [praca habilitacyjna], Warszawa 2012: 171-173.

8. Mandava A, Ravuri PR, Konathan R: High-resolution ultrasound imaging of cutaneous lesions. Indian J Radiol Imaging 2013; 23: 269-277.

9. Mlosek RK, Malinowska S, Sikora M, Debowska RM, Stępień A, Czekaj K et al:: The use of high frequency ultrasound imaging in skin moisturization measurment. Skin Res Technol 2013; 19: 169-175.

10. Mlosek RK, Malinowska S: Application of high-frequency ultrasound in closing small blood vessels. J Ultrason 2014; 14: 320-327.

11. Migda MS, Migda M, Migda B, Słapa RZ, Mlosek RK: Feasiblity of using high-frequency skin ultrasound (HFSU) in vulvar skin assessment - initial report with the description of HFSU anatomy. Ginekol Pol 2016; 87: 19-25.

12. Serup J: Characterization of contact dermatitis and atopy using bioengineering techniques. A survey. Acta Derm Venereol Suppl (Stockh) 1992; 177: 14-25.

13. Hoffmann K, Dirschka T, Schwarze H, el-Gammal S, Matthes U, Hoffmann A et al.: $20 \mathrm{MHz}$ sonography, colorimetry and image analysis in the evaluation of psoriasis vulgaris. J Dermatol Sci. 1995; 9: 103-110.

14. Polańska A, Dańczak-Pazdrowska A, Silny W, Woźniak A, Maksin K, Jenerowicz D et al.: Comparison between high-frequency ultrasonogra- layers and measurement of its thickness. These features of HFUS have been confirmed in the evaluation of the vulva, vagina and cervix in this study. This method confirms the layered structure of the vulvar skin and vaginal walls as well as the echostructure of the cervix. It helps visualize vulvar skin appendages. HFUS offers an objective assessment of the normal structure of the vulva, vagina and cervix.

\section{Conflict of interest}

Authors do not report any financial or personal connections with other persons or organizations, which might negatively affect the contents of this publication and/or claim authorship rights to this publication.

phy (Dermascan C, version 3) and histopathology in atopic dermatitis. Skin Res Technol 2013; 19: 432-437.

15. Kędzia H (ed.): Nowotwory narządów płciowych kobiety. OWN, Poznań 1997: 201-204, 205-217.

16. Smith CV, Anderson JC, Matamoros A, Rayburn WF: Transvaginal sonography of cervical with and length during pregnancy. J Ultrasund Med 1992; 11: 465-467.

17. Jackson GM, Ludmir J, Bader TJ: The accuracy of digital examination and ultrasound in the evaluation of cervical length. Obstet Gynecol 1992; 79: 214-218.

18. Timor-Trisch IE, Goldstein SR: Ultrasonografia w ginekologii. Dębski R (ed.), Elsevier Urban \& Partner, Wrocław 2008: 55-56.

19. Mayeaux EJ Jr, Thomas Cox J: Modern Colposcopy. Textbook \& Atlas Third Edition. Lippincott Williams \& Wilkins, 2012: 14-21; 24-26.

20. Levi CS, Holt S.C, Lyons EA, Lindsay DJ, Dashefsky SM.: Normal anatomy of the female pelvis. In: Callen PW (ed.): Ultrasonography in Obstetrics and Gynecology. Saunders Philadelphia 2000: 781-813.

21. Walker DK, Salibian RA, Salibian AD, Belen KM, Palmer SL: Overlooked diseases of the vagina: a directed anatomic-pathologic approach for imaging assessment. Radilograpics 2011; 31: 1583-1598.

22. Johnson SC, Yegul NT, Balcacer P: Sonovaginography: a useful technique in the assessment of the lower genital tract. J Ultrasound Med 2017; 36: 1917-1933.

23. Spaczyński M.red: Ultrasonografia w położnictwie i ginekologii. PZWL.1989: 32-34.

24. Merz E.: Diagnostyka ultrasonograficzna w ginekologii i położnictwie. Tom 1. Urban \& Partner, Wrocław 1997: 41-44.

25. Hamed ST, Mansour SM: Surface transperineal ultrasound and vaginal abnormalities: applications and strengths. Br J Radiol 2017; doi: 10.1259/bjr.20170326.

26. Varma TR, Patel RH, Pilai U: Ultrasonic assessment of cervix in normal pregnancy. Acta Obstet Gynecol Scand 1986; 65: 229-233.

27. Brown MA, Mattrey RF, Stamato S, Sirlin CB: MRI of the female pelvis using vaginal gel. AJR Am J Roentgenol 2005; 185: 1221-1227.

28. Bermejo C, Martínez-Ten P, Recio M, Ruiz-López D, Illescas T: Threedimensional ultrasound and magnetic resonance imaging assessment of cervix and vagina in women with uterine malformations. Ultrasound Obstet Gynecol 2014; 43: 336-345. 